NASA CONTRACTOR RE POR T

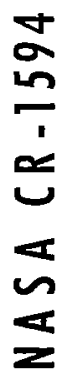

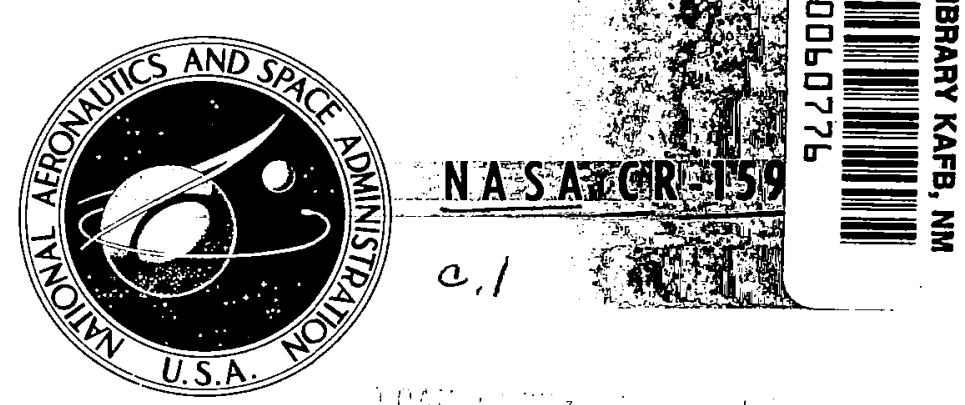

\title{
DECISION-MAKING IN A FUZZY ENVIRONMENT
}

by R. E. Bellman and L. A. Zadeh

Prepared by

UNIVERSITY OF CALIFORNIA

Berkeley, Calif. 94720

for

NATIONAL AERONAUTICS AND SPACE ADMINISTRATION - WASHINGTON, D. C. - MAY 1970 
By R. E. Bellman and L. A. Žadeh

Issued by Originator as Report No. ERL-69-8

Prepared under Grant No. NGL-05-003-016 by

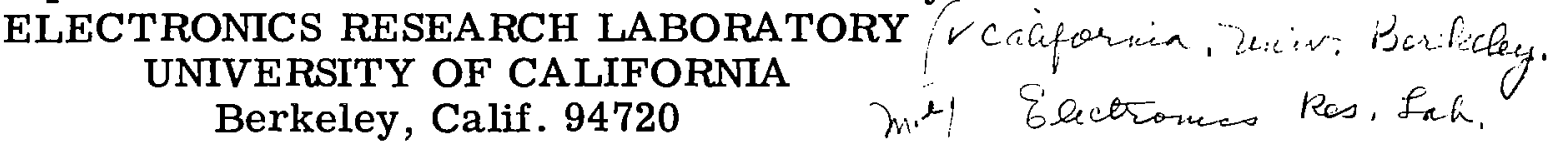

for

\section{NATIONAL AERONAUTICS AND SPACE ADMINISTRATION}





\begin{abstract}
By decision-making in a fuzzy environment is meant a decision process in which the goals and/or the constraints, but not necessarily the system under control, are fuzzy in nature. This means that the goals and/or the constraints constitute classes of alternatives whose boundaries are not sharply defined.
\end{abstract}

An example of a fuzzy constraint is: "The cost of A should not be substantially higher than $\alpha, "$ where $\alpha$ is a specified constant. Similarly, an example of a fuzzy goal is: "x should be in the vicinity of $x_{0}$," where $x_{0}$ is a constant. The underlined words are the sources of fuzziness in these examples.

Fuzzy goals and fuzzy constraints can be defined precisely as fuzzy sets in the space of alternatives. A fuzzy decision, then, may be viewed as an intersection of the given goals and constraints. A maximizing decision is defined as the set of points in the space of alternatives at which the membership function of a fuzzy decision attains its maximum value.

The use of these concepts is illustrated by examples involving multistage decision processes in which the system under 
control is elther deterministic or stochastic. By using dynamic programing, the determination of a maximizing decision is reduced to the solution of a system of functional equations. A reverse-flow technique is described for the solution of a functional equation arising in connection with a decision process in which the termination time is defined implicitly by the condition that the process stops when the system under control enters a specifled set of states in its state space. 
1. INTRODUCTION 1

2. A BRIEF INTRODUCTION TO FUZZY SETS 5

3. FUZZY GOALS, CONSTRAINTS AND DECISIONS 15

4. MULTISTAGE DECISION PROCESSES 25

5. STOCHASTIC SYSTEMS IN A FUZZY ENVIRONMENT 31

6. SYSTEMS WITH IMPLICITLY DEFINED TERMINATION TIME 36

7. CONCLUDING REMARKS

REFERENCES

FIGURES 


\section{INTRODUCTION}

Much of the decision-making in the real world takes place in an environment in which the goals, the constraints and the consequences of possible actions are not known precisely. To deal quantitatively with imprecision, we usually employ the concepts and techniques of probability theory and, more particularly, the tools provided by decision theory, control theory and information theory. In so doing, we are tacitly accepting the premise that imprecision - whatever its nature - can be equated with randomness. This, in our view, is a questionable assumption.

Specifically, our contention is that there is a need for differentiation between randomness and fuzziness, with the latter being a major source of imprecision in many decision processes. By fuzziness, we mean a type of imprecision which is associated with the use of fuzzy sets, ${ }^{1,2}$ that is, classes in which there is no sharp transition from membership to non-membership. For example, the class of green objects is a fuzzy set. So are the classes of objects characterized by such commonly used adjectives as large, small, substantial, significant, important, serious, simple, accurate, approximate, etc. Actually, in sharp contrast to the notion of a class or a set in mathematics, most of the classes in the real world do not have crisp boundaries which separate those objects which belong to the classes in question from those which do not. In this connection, it is important to note that, in the discourse between humans, statements such as "John is several inches taller 
than Jim," "x is much larger than $y, "$ "Corporation $x$ has a bright future," "the stock market has suffered a sharp decline," convey information despite the fuzziness of the meaning of the underlined words. In fact, It may be argued that the main distinction between human intelligence and machine intelligence lies in the ability of humans -- an ability which present-day computers do not possess -- to manipulate fuzzy concepts and respond to fuzzy instructions.

What is the distinction between randomness and fuzziness? Essentially, randomness has to do with uncertainty concerning membership or non-membership of an object in a non-fuzzy set. Fuzziness, on the other hand, has to do with classes in which there may be grades of membership intermediate between full membership and non-membership. To illustrate the point, the fuzzy assertion "Corporation $\mathrm{X}$ has a modern outlook" is Imprecise by virtue of the fuzziness of the terms "modern outlook." On the other hand, the statement "The probability that Corporation $X$ is operating at a loss is $0.8, "$ is a measure of the uncertainty concerning the membership of Corporation $\mathrm{X}$ in the non-fuzzy class of corporations which are operating at a loss.

Reflecting this distinction, the mathematical techniques for dealing with fuzziness are quite different from those of probability theory. They are simpler in many ways because to the notion of probability measure in probability theory corresponds the simpler notion of membership function in the theory of fuzziness. Furthermore, the cor- 
respondents of $a+b$ and $a b$, where $a$ and $b$ are real numbers, are the simpler operations $\operatorname{Max}(a, b)$ and $\operatorname{Min}(a, b)$. For this reason, even in those cases in which fuzziness in a decision process can be stmulated by a probabilistic model, it is generally advantageous to deal with it through the techniques provided by the theory of fuzzy sets rather than through the employment of the conceptual framework of probability theory.

Decision processes in which fuzziness enters in one way or another can be studied from many points of view. $3,4,5$ In the present note, our main concern is with introducing three basic concepts: fuzzy goal, fuzzy constraint and fuzzy decision, and exploring the application of these concepts to multistage decision processes in which the goals or the constraints may be fuzzy, while the system under control may be either deterministic or stochastic - but not fuzzy. This, however, is not an intrinsic restriction on the applicability of the concepts and techniques described in the following sections.

Roughly speaking, by a fuzzy goal we mean an objective which can be characterized as a fuzzy set in an appropriate space. To 11lustrate, a simple example of a fuzzy goal involving a real-valued vartable $x$ would be: "x should be substantlally larger than 100." Simflarly, a simple example of a fuzzy constraint would be: "x should be approximately in the range 20-25." The sources of fuzziness in these statements are the underlined words. 
A less trivial example is provided by a deterministic discrete-time system characterized by the state equations

$$
x_{n+1}=x_{n}+u_{n} \quad, n=0,1,2, \ldots
$$

where $x_{n}$ and $u_{n}$ denote, respectively, the state and input at time $n$ and in which for simplicity $x_{n}$ and $u_{n}$ are assumed to be real-valued. Here a fuzzy constraint on the input may be

$$
-1 \leq \mathrm{u}_{\mathrm{n}} \leq 1
$$

where the wavy bar under a symbol plays the role of a fuzzifier, that is, a transformation which takes a non-fuzzy set into a fuzzy set which is approximately equal to $i t$. In this instance, $u_{n} \leq 1$, would read " $u_{n}$ should be approximately less than or equal to 1 , and the effect of the fuzzifier is to transform the non-fuzzy set $-1 \leq u_{n} \leq 1$ into a fuzzy set $-1 \leq u_{n} \leq 1$. The way in which the latter set can be given a precise meaning will be discussed in Section 2 .

Assume that the fuzzy goal is to make $x_{3}$ approximately equal to 5 , stariing with the initial state $x_{0}=1$. Then, the problem is to find a sequence of inputs $u_{0}, u_{1}, u_{2}$ which w111 realize the specified goal as nearly as possible, subject to the specified constraints on $u_{0}, u_{1}$, $\mathbf{u}_{2} \cdot$

In what follows, we shall consider in greater detail a few representative problems of this type. It should be stressed that our limited objective in the present paper is to draw attention to problems Involving multistage decision processes in a fuzzy environment and 
suggest tentative ways of attacking them, rather than to develop a general theory of dectsion processes in which fuzziness and randomness may enter in a variety of ways and combinations. In particular, we shall not concern ourselves with the application to decision-making of the concept of a fuzzy algorithm ${ }^{3}$ - a concept which may be of use in problems which are less susceptible to quantitative analysis than those considered in the sequel.

For convenience of the reader, a brief summary of the basic properties of fuzzy sets is provided in the following section.

\section{A Brief Introduction to Fuzzy Sets}

Informa11y, a fuzzy set is a class of objects in which there is no sharp boundary between those objects that belong to the class and those that do not. A more precise definition may be stated as follows.

Definition. Let $X=\{x\}$ denote a collection of objects (points) denoted generically by $x$. Than a fuzzy set $A$ in $X$ is a set of ordered pairs

$$
A=\left\{\left(x, \mu_{A}(x)\right)\right\} \quad, \quad x \in X
$$

where $\mu_{A}(x)$ is termed the grade of membership of $x$ in $A$, and $\mu_{A}: X \rightarrow M$ is a function from $X$ to a space $M$ called the membership space. When $M$ contains only two points, 0 and $1, A$ is non-fuzzy and its membership 
function becomes identical with the characteristic function of a nonfuzzy set.

In what follows, we shall assume that $M$ is the interval $[0,1]$, with 0 and 1 representing, respectively, the lowest and highest grades of membership. (More generally, $M$ can be a partially ordered set or, more particularly, a lattice ${ }^{6,7}$.) Thus, our basic assumption is that a fuzzy set A - despite the unsharpness of its boundaries - can be defined precisely by assoclating with each object $x$ a number between 0 and 1 which represents its grade of membership in $A$.

Example Let $x=\{0,1,2, \ldots\}$ be the collection of non-negative integers. In this space, the fuzzy set A of "several objects" may be defined (subjectively) as the collection of ordered pairs

$$
A=\{(3,0.6),(4,0.8),(5,1.0),(6,1.0),(7,0.8),(8,0.6)\}
$$

with the understanding that in (2) we list only those pairs $\left(x, \mu_{A}(x)\right)$ in which $\mu_{A}(x)$ is positive.

Comment. It should be noted that in many practical situations the membership function, $\mu_{A}$, has to be estimated from partial information about it, such as the values which it takes over a finite set of sample points $\mathrm{x}_{1}, \ldots, \mathrm{x}_{\mathrm{N}}$. When $\mathrm{A}$ is defined incompletely - and hence only approximately in this fashion, we shall say that it is partially defined by exemp1i- 
fication. The problem of estimating $\mu_{A}$ from the knowledge of the set of pairs $\left(x_{1}, \mu_{A}\left(x_{1}\right)\right), \ldots\left(x_{N}, \mu_{A}\left(x_{N}\right)\right)$ is the problem of abstraction - a problem that plays a central role in pattern recognition. 8,9 we shall not concern ourselves with the solution of this problem in the present paper and will assume throughout - except where explicitly stated to the contrary - that $\mu_{A}(x)$ is given for all $x$ in $x$.

For notational purposes, it is convenient to have a device for indicating that a fuzzy set A is obtained from a non-fuzzy set $\bar{A}$ by fuzzlfying the boundaries of the latter set. For this purpose, we sha11 employ a wavy bar under a symbol (or symbols) which define $\bar{A}$. For example, if $A$ is the set of real numbers between 2 and $5,1 . e ., \bar{A}=\{x \mid$ $2 \leq x \leq 5\}$, then $A=\{\dot{x} \mid 2 \leq x \leq 5\}$ is a fuzzy set of real numbers which are approximately between 2 and 5. Simflarly, $A=\{x \mid x=5\}$ or simply 5 will denote the set of numbers which are approximately equal to 5 . The symbol $\sim$ will be referred to as a fuzzifier.

We turn next to the definition of several basic concepts which we shall need in later sections.

Normality: A fuzzy set $A$ is normal if and only if $\operatorname{Sup}_{x} \mu_{A}(x)=1$, that is, the supremum of $\mu_{A}(x)$ over $X$ is unity. A fuzzy set is subnormal if it is not normal. A non-empty subnormal fuzzy set can be normalized by dividing each $\mu_{A}(x)$ by the factor $\sup _{\mathbf{x}} \mu_{A}(x)$. (A fuzzy set $A$ is empty if and only if $\left.\mu_{A}(x) \equiv 0.\right)$ 
Equality: Two fuzzy sets are equal, written as $A=B$, if and only if $\mu_{A}=\mu_{B}$, that is, $\mu_{A}(x)=\mu_{B}(x)$ for all $x$ in $x$. (In the sequel, to simplify the notation we shall omit the argument $x$ when an equality or inequality holds for all values of $x$ in $x$. )

Containment: A fuzzy set A is contained in or is a subset of a fuzzy set $B$, written as $A \subset B$, if and only if $\mu_{A} \leq \mu_{B}$. In this sense, the fuzzy sat of very large numbers is a subset of the fuzzy set of large numbers.

Complementation: $A^{\prime}$ is said to be the complement of $A$ if and only if $\mu_{A^{\prime}}=1-\mu_{A^{\prime}}$ For example, the fuzzy sets: $A=\{\operatorname{tall}$ men $\}$ and $A^{\prime}=$ \{not tall men\} are complements of one another if the negation "not" is interpreted as an operation which replaces $\mu_{A}(x)$ with $1-\mu_{A}(x)$ for each $\mathrm{x}$ in $\mathrm{x}$.

Intersection: The intersection of $A$ and $B$ is denoted by $A \cap B$ and is defined as the largest fuzzy set contained in both $A$ and $B$. The membership function of $A \cap B$ is given by

$$
\mu_{A} \cap B(x)=\operatorname{Min}\left(\mu_{A}(x), \mu_{B}(x)\right), \quad x \in x
$$

where $\operatorname{Min}(a, b)=a$ if $a \leq b$ and $\operatorname{Min}(a, b)=b$ if $a>b$. In Infix form, using the conjunction symbol $\Lambda$ in place of Min, (3) can be written more simply as

$$
\mu_{\mathrm{A}} \cap \mathrm{B}=\mu_{\mathrm{A}} \wedge \mu_{\mathrm{B}}
$$


The notion of intersection bears a close relation to the notion of the connective "and." Thus, if $A$ is the class of tall men and $B$ is the class of fat men, then $A \cap B$ is the class of men who are both tall and fat.

Comment. It should be noted that our identification of "and" with (4) implies that we are interpreting "and" in a "hard" sense, that is, we do not allow any tradeoff between $\mu_{A}(x)$ and $\mu_{B}(x)$ so long as $\mu_{A}(x)>\mu_{B}(x)$. For example, if $\mu_{A}(x)=0.8$ and $\mu_{B}(x)=0.5$, then $\mu_{A} \cap B(x)=0.5$ so long as $\mu_{A}(x) \geq 0.5$. In some cases, a softer interpretation of "and" which corresponds to forming the algebraic product of $\mu_{A}(x)$ and $\mu_{B}(x)$ - rather than the conjunction $\mu_{A}(x) \wedge \mu_{B}(x)$ - may be closer to the intended meaning of "and." From the mathematical as well as practical points of view, the identification of "and" with $\wedge$ is preferable to its identification with the product, except where $\Lambda$ clearly does not express the sense in which one wants "and" to be interpreted. For this reason, in what follows "and" will be understood to be a hard "and" unless explicitly stated that it should be interpreted as a soft "and" (in the sense of corresponding to the algebraic product of membership functions).

Union: The notion of the union of $A$ of $B$ is dual to the notion of intersection. Thus, the union of $A$ and $B$, denoted as $A \cup B$, is defined as the smallest fuzzy set containing both $A$ and $B$. The membership function of $\mathrm{A} \cup \mathrm{B}$ is given by

$$
\mu_{A} \cup B(x)=\operatorname{Max}\left(\mu_{A}(x), \mu_{B}(x)\right), \quad x \in X
$$


where $\operatorname{Max}(a, b)=a$ if $a \geq b$ and $\operatorname{Max}(a, b)=b$ if $a<b$. In Infix form, using the disjunction symbol $v$ in place of Max, we can write (5) more simply as

$$
\mu_{A} \cup B=\mu_{A} \vee \mu_{B}
$$

As in the case of the intersection, the union of $A$ and $B$ bears a close relation to the connective "or." Thus, if $A=\{$ tall men $\}$ and $B=\{$ fat men $\}$, then $A \cup B=\{$ tall or fat men $\}$. Also, we can differentiate between a hard "or", which corresponds to (6), and a soft "or", which corresponds to the algebraic sum of $A$ and $B$, which is denoted by $A \oplus B$ and is defined by (9).

It is easy to verify that $U$ and $\cap$ are related to one another by the identity

$$
A \cup B=\left(A^{\prime} \cap B^{\prime}\right)^{\prime}
$$

Algebraic product: The algebraic product of $A$ and $B$ is denoted by $A B$ and is defined by

$$
\mu_{A B}(x)=\mu_{A}(x) \mu_{B}(x), x \varepsilon X
$$

Algebraic sum: The algebraic sum of $A$ and $B$ is denoted by $A \oplus B$ and is defined by 


$$
\mu_{A} \oplus B(x)=\mu_{A}(x)+\mu_{B}(x)-\mu_{A}(x) \mu_{B}(x), x \in x
$$

It is easy to verify that

$$
A \text { 且 } B=\left(A^{\prime} B^{\prime}\right)^{\prime} \text {. }
$$

Comment. It should be noted that the operations $v$ and $\Lambda$ are associative and distributive, over one another. On the other hand, (product) and (sum) are associative but not distributive.

Convexity and concavity. Let $A$ be a fuzzy set in $X=R^{n}$. Then $A$ is convex If and only if for every pair of points $(x, y)$ in $x$, the membership function of A satisfies the inequality

$$
\mu_{A}(\lambda x+(1-\lambda) y) \geq \operatorname{Min}\left(\mu_{A}(x), \mu_{A}(y)\right)
$$

. for $0 \leq \lambda \leq 1$. Dually, $A$ is concave if its complement $A^{\prime}$ is convex. It is easy to show that if $A$ and $B$ are convex, so is $A$ B. Dually, if $A$ and $B$ are concave, so is $A U_{B}$.

Relation. A fuzzy relation, $R$, in the product space $X x Y=\{(x, y)\}, x \in X$, $y \in Y$, is a fuzzy set in $X \times Y$ characterized by a membership function $\mu_{R}$ which associates with each ordered pair $(x, y)$ a grade of membership $\mu_{R}(x, y)$ in $R$. More generally, an $n$-ary fuzzy relation in a product space 
$x=x^{1} \times x^{2} \times \ldots . . x x^{n}$ is a fuzzy set in $x$ characterized by an n-variate membership function $\mu_{R}\left(x_{1}, \ldots, x_{n}\right), x_{1} \varepsilon x^{1}, 1=1, \ldots, n$.

Example. Let $X=Y=R^{I}$, where $R^{1}$ is the real line $(-\infty, \infty)$. Then $x>>y$ is a fuzzy relation in $R^{2}$. A subjective expression for $\mu_{R}$ in this case might be: $\mu_{R}(x, y)=0$ for $x \leq y ; \mu_{R}(x, y)=\left(1+(x-y)^{-2}\right)^{-1}$ for $x>y$.

Fuzzy sets induced by mappings. Let $f: X+Y$ be a mapping from $X=\{x\}$ to $Y=\{y\}$, with the image of $x$ under $f$ denoted by $y=f(x)$. Let $A$ be a fuzzy set in $X$. Then, the mapping $f$ induces a fuzzy set $B$ in $Y$ whose. membership function is given by

$$
\mu_{B}(y)=\operatorname{Sup}_{x \in f^{-1}(y)} \mu_{A}(x)
$$

where the supremum is taken over the set of points $f^{-1}(y)$ in $X$ which are mapped by $f$ into $y$.

Conditioned fuzzy sets: A fuzzy set $B(x)$ in $Y=\{y\}$ is conditioned on $x$ if its membership function depends on $x$ as a parameter. This dependence is expressed by $\mu_{B}(y \mid x)$.

Suppose that the parameter $\mathrm{x}$ ranges over a space $\mathrm{X}$, so that to each $X$ in $X$ corresponds a fuzzy set $B(X)$ in $Y$. Thus, we have a mapping - characterized by $\mu_{B}(y \mid x)$ - from $X$ to the space of fuzzy sets 
in $Y$. Through this mapping, any given fuzzy set $A$ in $X$ induces ä fuzzy set $B$ in $Y$ which is defined by

$$
\mu_{B}(y)=\sup _{x} \operatorname{Min}\left(\mu_{A}(x), \mu_{B}(y \mid x)\right)
$$

where $\mu_{A}$ and $\mu_{B}$ denote the membership functions of $A$ and $B$, respectively. In terms of $\Lambda$ and $v$ (13) may be written more simply as

$$
\mu_{B}(y)=\underset{x}{v}\left(\mu_{A}(x) \wedge \mu_{B}(y \mid x)\right)
$$

Note that this equation is analogous - but not equivalent - to the expression for the marginal probability distribution of the joint distribtion of two random variables, with $\mu_{B}(y \mid x)$ playing a role analogous to that of a conditional distribution.

Decomposability: Let $X=\{x\}, Y=\{y\}$ and let $C$ be a fuzzy set in the product space $Z=X X Y$ defined by a membership function $\mu_{c}(x, y)$. Then $C$ is decomposable along $X$ and $Y$ if and only if $C$ admits of the representation

$$
\mathrm{C}=\mathrm{A} \cap \mathrm{B}
$$

or equivalently

$$
\mu_{c}(x, y)=\mu_{A}(x) \wedge \mu_{B}(y)
$$


where $A$ and $B$ are fuzzy sets with membership functions of the form $\mu_{A}(x)$ and $\mu_{B}(y)$, respectively. (Thus, $A$ and $B$ are cylindrical fuzzy sets in Z.) The same holds for a fuzzy set in the product of any finite number of spaces.

Probability of fuzzy events: Let $P$ be a probability measure on $R^{n}$. A fuzzy event ${ }^{10} A$ in $R^{\mathfrak{n}}$ is defined to be a fuzzy subset $A$ of $R^{n}$ whose membership function, $\mu_{A}$, is measurable. Then, the probability of $A$ is defined by the Lebesgue-Stieltjes integral.

$$
P(A)=\int_{R^{n}} \mu_{A}(x) d P
$$

Equivalently,

$$
P(A)=E \mu_{A}
$$

where $E$ denotes the expectation operator. In the case of a non-fuzzy set, (16) reduces to the conventional definition of the probability of a nonfuzzy event.

This concludes our brief introduction to some of the basic concepts relating to fuzzy sets. In the following section, we shall use these concepts as a basis for defining the central notions of goal, constraint and decision in a fuzzy environment. 


\section{Fuzzy Goals, Constraints and Decisions}

In the conventional approach to decision-making, the principal ingredients of a decision process are (a) A set of alternatives; (b) a set of constraints on the cholce between different alternatives; and (c) a performance function which associates with each alternative the gain (or loss) resulting from the choice of that alternative.

When we view a decision process from the broader perspective of decision-making in a fuzzy environment, a different and perhaps more natural conceptual framework suggests itself. The most important feature of this framework is its symmetry with respect to goals and constraints - a symmetry, which erases the differences between them and makes it possible to relate in a relatively simple way the concept of a decision to those of the goals and constraints of a dectsion process.

More specifically, let $X=\{x\}$ be a given set of alternatives. Then, a fuzzy goal or simply a goal, $G$, in $X$ will be identified with a given fuzzy set $G$ in $X$. For example, if $X=R^{1}$ (the real 1 ine), then the fuzzy goal expressed in words as " $x$ should be substantially larger than 10" might be represented by a fuzzy set in $R^{1}$ whose membership function is (subjectively) given by

$$
\mu_{G}(x)=0, \quad x<10
$$




$$
=\left(1+(x-10)^{-2}\right)^{-1}, x \geq 10
$$

Similarly, the goal " $x$ should be in the vicinity of 15 " might be represented by a fuzzy set whose membership function is of the form

$$
\mu_{G}(x)=\left(1+(x-15)^{-4}\right)^{-1}
$$

Note that both of these sets axe convex in the sense of (11).

In the conventional approach, the performance function associated with a decision process serves to define a linear ordering on the set of alternatives. Clearly, the membership function, $\mu_{G}(x)$, of a fuzzy goal serves the same purpose* and, in fact, may be derived from a given performance function by a normalization which leaves the linear ordering unaltered. In effect, such normalization provides a common denominator for the various goals and constraints and thereby make's it possible to treat them alike. This, as we shall see, is one of the significant advantages of regarding the concept of a goal - rather than that of a performance function - as one of the princlpal components of a conceptual framework for decision-making in a fuzzy environment.

* Assuming, of course, that $\mu_{G}$ takes values in a Iinearly ordered set. 
In a simflar manner, a fuzzy constraint or simply a constraint, $C$, in $X$ is defined to be a fuzzy set in $X$. For example, in $\mathrm{R}^{1}$, the constraint " $\mathrm{x}$ should be approximately between 2 and $10, "$ could be represented by a fuzzy set whose membership function might be of the form

$$
\mu_{c}(x)=\left(1+a(x-6)^{-m}\right)^{-1}
$$

where $a$ is a positive number and $m$ is a positive even integer chosen in such a way as to reflect the sense in which the approximation to the interval $[2,10]$ is to be understood. For example, if we set $m=4$ and $a=$ $5^{-4}$, then at $x=2$ and $x=10$ we have approximately $\mu_{c}(x)=0.8$, while at $x=1$ and $x=11, \mu_{c}(x)=0.5$; and at $x=0$ and $x=12, \mu_{c}(x)$ is approximately equal to 0.3 .

An important aspect of the above definitions of the concepts of goal and constraint is that both are defined as fuzzy sets in the space of alternatives and thus, as will be elaborated upon below, can be treated identically in the formulation of a decision. By contrast, in the conventional approach to decision-making, a constraint set is taken to be a non-fuzzy set in the space of alternatives $x$, whereas a performance function is a function from $X$ to some other space. Nevertheless, even in the case of the conventional approach, the use of Lagrangian multipliers and penalty functions makes it apparent that there is an intrinsic similarity between performance functions and con- 
straints*. This similarity - indeed identity - is made explicit in our formulation.

As an illustration, suppose that we have a fuzzy goal G and a fuzzy constraint $C$ expressed as follows:

G: $x$ should be substantially larger than 10 , with $\mu_{G}(x)$ gIven by (17) and

C: $x$ should be in the vicinity of 15 , with $\mu_{c}(x)$ expressed by (18).

Note that $G$ and $C$ are connected to one another by the connective and. Now, as was pointed out in Sec.2, and corresponds to the intersection of fuzzy sets. This implies that in the example under consideration the combined effect of the fuzzy goal $G$ and the fuzzy constraint $C$ on the choice of alternatives may be represented by the intersection $G \cap C$. The membership function of the intersection is given by

$$
\mu_{G} \cap \mathrm{C}(\mathrm{x})=\mu_{\mathrm{G}}(\mathrm{x}) \wedge \mu_{\mathrm{c}}(\mathrm{x})
$$

or more explicit1y

$$
\mu_{G \cap X}(x)=\operatorname{Min}\left(\left(1+(x-10)^{-2}\right)^{-1},\left(1+(x-15)^{-4}\right)^{-1}\right) \text { for } x \geq 10
$$

*For a very thorough discussion of these points see Ref. 12. 
Note that $G \cap C$ is a convex fuzzy set since both $G$ and $C$ are convex fuzzy sets.

\begin{abstract}
Turning to the concept of a decision, we observe that, intuitively, a decision is basically a cholce or a set of choices drawn from the available alternatives. The preceding example suggests that a fuzzy deciston or simply a decision be defined as the fuzzy set of alternatives resulting from the intersection of the goals and constraints. We formalize this idea in the following definition.
\end{abstract}

Definition. Assume that we are given a fuzzy goal G and a fuzzy constraint $C$ in a space of alternatives $X$. Then, $G$ and $C$ combine to form a decision, $D$, which is a fuzzy set resulting from intersection of $G$ and $C$. In symbols,

$$
\mathrm{D}=\mathrm{G} \cap \mathrm{C}
$$

and corresponding1y

$$
\mu_{D}=\mu_{G} \wedge \mu_{C}
$$

The relation between $G, C$ and $D$ is depicted in Fig.1. 
More generally, suppose that we have $n$ goals $G_{1}, \ldots, G_{n}$ and $m$ constraints $C_{1} \ldots, C_{m}$. Then, the resultant decision is the intersection of the given goals $G_{1}, \ldots, G_{n}$ and the given constraints $C_{1}, \ldots$, $\mathrm{C}_{\mathrm{m}}$. That is,

$$
D=G_{1} \cap G_{2} \cap \ldots \cap G_{n} \cap C_{1} \cap C_{2} \cap \ldots \cap C_{m}
$$

and correspondingly

$$
\mu_{D}=\mu_{G_{1}} \wedge \mu_{G_{2}} \wedge \ldots \mu_{G_{n}} \wedge \mu_{C_{1}} \wedge \mu_{C_{2}} \wedge \ldots \wedge \mu_{C_{m}}
$$

Note that in the above definition of a decision, the goals and the constraints enter into the expression for $D$ in exactly the same way. This is the basis for our earlier statement concerning the identity of the roles of goals and constraints in our formulation of decision processes in a fuzzy environment.

Comment. The definition of a decision as the intersection of the goals and constraints, reflects our interpretation of "and" in the "hard" sense of (4). If the interpretation of "and" is left open, we shall say that a decision - viewed as a fuzzy set - is a confluence of the goals and the constraints. Thus, "confluence" acquires the meaning of "intersection" when "and" is interpreted in the sense of (4); the meanIng of "algebraic product" when "and" is interpreted in the sense of 
(8); and may be assigned other concrete meanings when a need for a speclal interpretation of "and" arises. In short, a broad definition of the concept of decision may be stated as:

$$
\text { Decision }=\text { Confluence of Goals and Constraints }
$$

As an illustration of (21), we shall consider a very simple example in which $X=\{1,2, \ldots, 10\}$ and $G_{1}, G_{2}, C_{1}$ and $C_{2}$ are defined below.

\begin{tabular}{l|rrrrrrrrrr}
$\mathrm{x}$ & 1 & 2 & 3 & 4 & 5 & 6 & 7 & 8 & 9 & 10 \\
\hline$\mu_{\mathrm{G}_{1}}$ & 0 & 0.1 & 0.4 & 0.8 & 1.0 & 0.7 & 0.4 & 0.2 & 0 & 0 \\
$\mu_{\mathrm{G}_{2}}$ & 0.1 & 0.6 & 1.0 & 0.9 & 0.8 & 0.6 & 0.5 & 0.3 & 0 & 0 \\
$\mu_{\mathrm{C}_{1}}$ & 0.3 & 0.6 & 0.9 & 1.0 & 0.8 & 0.7 & 0.5 & 0.3 & 0.2 & 0.1 \\
$\mu_{\mathrm{C}_{2}}$ & 0.2 & 0.4 & 0.6 & 0.7 & 0.9 & 1.0 & 0.8 & 0.6 & 0.4 & 0.2
\end{tabular}

Forming the conjunction of $\mu_{G_{1}}, \mu_{G_{2}}, \mu_{C_{1}}$ and $\mu_{C_{2}}$, we obtain the following table of values for $\mu_{D}(x)$

\begin{tabular}{l|rrrrrrrrrr}
$\mathrm{x}$ & 1 & 2 & 3 & 4 & 5 & 6 & 7 & 8 & 9 & 10 \\
\hline$\mu_{\mathrm{D}}$ & 0 & 0.1 & 0.4 & 0.7 & 0.8 & 0.6 & 0.4 & 0.2 & 0 & 0
\end{tabular}

Thus the decision in this case is the fuzzy set

$$
D=\{(2,0.1),(3,0.4),(4,0.7),(5,0.8),(6,0.6),(7,0.4),(8,0.2)\}
$$


Note that no $x$ in $x$ has full (that is, unity grade) membership in $D$. This reflects, of course, the fact that the specified goals and constraints conflict with one another, ruling out the existence of an alternative which fully satisfles all of them.

The concept of a decision as a fuzzy set in the space of alternatives may appear at first to be somewhat artificial. In fact it is quite natural, since a fuzzy decision may be viewed as an Instruction whose fuzziness is a consequence of the imprecision of the given goals and constraints. Thus, in our example, $G_{1}, G_{2}, C_{1}$ and $C_{2}$ may be respectively expressed in words as: "x should be close to 5, " "x should be close to $3, "$ "x should be close to 4 " and "x should be close to 6". The decision, then, is to choose $\mathrm{x}$ to be close to 5 . The exact meaning of "close" in each case is given by the values of the corresponding membership function.

How should a fuzzy instruction such as " $x$ should be close to $5^{\prime \prime}$ be executed? Although there does not appear to be a universally valid answer to questions of this type, * it is reasonable in many instances to choose that $x$ or $x^{\prime}$ 's wich have maximal grade of membership in D. In the case of our example, this would be $x=5$.

* The execution of fuzzy instructions is discussed in Ref. 3 . 
More generally, let $D$ be a fuzzy decision and let $D^{M}$ be its core, that is, the non-fuzzy set of points in $X$ at which the maximum of $\mu_{D}(x)$ over $X$, if it exists, is attalned. Then, we shall say that any $x$ in $D^{M}$ constitutes a maximizing dectsion. In other words, a maximizing decision is simply any alternative in $x$ which maximizes $\mu_{D}(x), e \cdot g \cdot, x=5$ in the foregoing example. Note that in $R^{n}$ a suffictent condition for the uniqueness of a maximfing decision is that $D$ be a strongly convex fuzzy set. *

In defining a fuzzy decision $D$ as the intersection - or more generally, as the confluence - of the goals and constraints, we are tacitly assuming that all of the goals and constraints that enter into D are, in a sense, of equal importance. These are some situations, however, in which some of the goals and perhaps some of the constraints are of greater importance than others. In such cases, D might be expressed as a convex combination of the goals and the constraints, with the weighting coefficients reflecting the relative importance of the constituent terms. More explicitly, we may express $\mu_{D}(x)$ as

$$
\mu_{D}(x)=\sum_{i=1}^{n} \alpha_{i}(x) \mu_{G_{i}}(x)+\sum_{j=1}^{m} B_{j}(x) \mu_{C_{j}}(x)
$$

* A fuzzy set is strongly convex if it is convex and its membership function is unimodal. 
where the $\alpha_{1}$ and $\beta_{j}$ are membership functions such that

$$
\sum_{i=1}^{n} \alpha_{i}(x)+\sum_{j=1}^{m} \beta_{j}(x) \equiv 1
$$

Subject to this constraint, then, the values of $\alpha_{i}(x)$ and $\beta_{j}(x)$ can be chosen in such a way as to reflect the relative importance of $G_{1}, \ldots, G_{n}$ and $C_{1}, \ldots, C_{m}$. In particular, if $m=n=1$, it is easy to verify that (22) can generate any fuzzy set which is contained in $G U_{C}$ and contains $G \cap C$. Note that (22) resembles the familiar artifice of transforming a vector-valued criterion into a scalar-valued criterion by forming a linear combination of the components of the vector-valued objective function.

So far, we have restricted our attention to situations in which the goals and the constraints are fuzzy sets in $\mathrm{x}$, the space of alternatives. A more general case which is of practical interest is one in which the goals and the constraints are fuzzy sets in different spaces. Specifically, let $f$ be a mapping from $X=\{x\}$ to $Y=\{y\}$, with $x$ representing an input (cause) and $y, y=f(x)$, representing the corresponding output (effect).

Suppose that the goals are defined as fuzzy sets $G_{1}, \ldots$, $G_{n}$ in $Y$ while the constraints $C_{1}, \ldots, C_{m}$ are defined as fuzzy sets in $X$. Now, given a fuzzy set $G_{i}$ in $Y$, one can readily find a fuzzy set $G_{i}{ }^{*}$ in 
$X$ which induces $G_{1}$ in $Y$. Speciffcally, the membership function of $G_{I}$ * is given by the equality

$$
\mu_{G_{1}} *(x)=\mu_{G_{1}}(f(x)) \quad, 1=1, \ldots, n
$$

The decision $D$; then, can be expressed as the intersection of $G_{1}^{*}, \ldots, G_{n}^{*}$ and $C_{1}, \ldots, C_{m}$. Using $(23)$, we can express $\mu_{D}(x)$ more explicitly as

$\mu_{D}(x)=\mu_{G_{1}}(f(x)) \wedge \ldots \wedge \mu_{G_{n}}(f(x)) \wedge \mu_{C_{1}}(x) \wedge \ldots \wedge \mu_{C_{m}}(x)$

where $f: X \rightarrow Y$. In this way, the case where the goals and the constraints are defined as fuzzy sets in different spaces can be reduced to the case where they are defined in the same space. We shall find (24) of use in the analysis of multistage decision processes in the following section.

\section{Multistage Deciston Processes}

As an application of the concepts introduced in the preceding sections, we shall consider a few basic types of problems involving multistage decision-making in a fuzzy environment. It should be stressed that, in what follows, our main purpose is to illustrate the use of the concepts of fuzzy goal, fuzzy constraint and fuzzy decision, rather than to develop a general theory of multistage decision processes in 
which fuzziness enters in one way or another.

For simplicity we shall assume that the system under control, A, is a time-invariant finite-state deterministic system in which the state, $x_{t}$, at time $t, t=0,1,2, \ldots$, ranges over a finite set $x=$ $\left\{\sigma_{1}, \ldots, \sigma_{n}\right\}$, and the input $u_{t}$, ranges over a finite set $U=\left\{\alpha_{1}, \ldots, \alpha_{m}\right\}$. The temporal evolution of $A$ is described by the state equation

$$
x_{t+1}=f\left(x_{t}, u_{t}\right), t=0,1,2, \ldots
$$

in which $f$ is a given function from $X x U$ to $X$. Thus, $f\left(x_{t}, u_{t}\right)$ represents the successor state of $x_{t}$ for input $u_{t}$. Note, that if $f$ is a random function, then $A$ is a stochastic system whose state at time $t+1$ is a probability distribution over $X, P\left(x_{t+1} \mid x_{t}, u_{t}\right)$, which is conditioned on $x_{t}$ and $u_{t}$. Analogously, if $f$ is a fuzzy function, then $A$ is a fuzzy system whose state at time $t+1$ is a fuzzy set conditioned on $x_{t}$ and $u_{t}$, which means that it is characterized by a membership function of the form $\mu\left(x_{t+1} \mid x_{t}, u_{t}\right){ }^{*}$ Since we will not be concerned with such systems in the sequel, it will be understood that $f$ is non-fuzzy unless explicitly stated to the contrary.

We assume that at each time $t$ the input is subjected to a fuzzy constraint $c^{t}$, which is a fuzzy set in $U$ characterized by a membership function $\mu_{t}\left(u_{t}\right)$. Furthermore, we assume that the goal is a

* It should be noted that when we speak of a fuzzy environment, we mean that the goals and/or the constraints are fuzzy, but not necessarily the system which is under control. 
fuzzy set $G^{N}$ in $X$, which is characterized by a membership function $\mu_{G} N\left(x_{N}\right)$, where $N$ is the time of termination of the process. These assumptions are common to most of the problems considered in the sequel.

Problem 1. In this case, the system is assumed to be characterized by (25), with $f$ a given non-random function. The termination time $N$ is assumed to be fixed and specified. The initial state, $x_{0}$, is assumed to be given. The problem is to find a maximizing decision.

Applying (20), the decision - viewed as a decomposable fuzzy set in $\mathrm{U} \times \mathrm{U} \times \ldots \times \mathrm{U}$, may be expressed at once as

$$
D=c^{\circ} \cap c^{1} \cap i \ldots \cap c^{i-1} \cap \bar{G}^{N}
$$

where $\bar{G}^{N}$ is the fuzzy set in $U \times U \times \ldots \times U$ which induces $G^{N}$ in $X$. More explicitly, in terms of membership functions, we have

$\mu_{D}\left(u_{0}, \ldots, u_{N-1}\right)=\mu_{0}\left(u_{0}\right) \wedge \ldots \mu_{N-1}\left(u_{N-1}\right) \Lambda_{G} \mu_{N}\left(x_{N}\right)$

where $x_{\mathrm{N}}$ is expressible as a function of $x_{0}$ and $u_{0}, \ldots, u_{N-1}$ through the iteration of (25). 
Our problem, then, is to find a sequence of inputs $u_{0}, \ldots, u_{N-1}$ which maximizes $\mu_{D}$ as given by (27). As is usually the case in multistage processes, it is expedient to express the solution in the form

$$
u_{t}=\pi_{t}\left(x_{t}\right) \quad, t=0,1,2, \ldots, N-1,
$$

where $\pi_{t}$ is a policy function. Then, we can employ dynamic programming to give us both the $\pi_{t}$ and the maximizing dectsion $u_{0}^{M}, \ldots, u_{N-I}^{M}$.

More specifically, using (26) and (25), we can write

$$
\begin{aligned}
& \mu_{D}\left(u_{0}^{M}, \ldots, u_{N-1}^{M}\right)=\operatorname{Max}_{u_{0}, \ldots, u_{N-2}} \operatorname{Max}_{N-1}^{u_{N}}\left(\mu_{o}\left(u_{o}\right) \wedge \ldots \mu_{N-2}\left(u_{N-2}\right) \wedge\right. \\
& \mu_{N-1}\left(u_{N-1}\right) \mu_{G} N^{N}\left(f\left(x_{N-1}, u_{N-1}\right)\right)
\end{aligned}
$$

Now, if $\gamma$ is a constant and $y$ is any function of $u_{\mathbb{N}-1}$, we have the identity

$$
\operatorname{Max}_{u_{N-1}}\left(\gamma \wedge_{g}\left(u_{N-1}\right)\right)=\gamma \wedge \operatorname{Max}_{u_{N-1}}\left(u_{N-1}\right)
$$

Consequently, (28) may be rewritten as 


$$
\begin{gathered}
\mu_{D}\left(u_{0}^{M}, \ldots, u_{N-1}^{N}\right)=\operatorname{Max}_{u_{0}, \ldots, u_{N-1}}\left(\mu_{0}\left(u_{0}\right) \wedge \ldots \Lambda_{\mu}\left(u_{N-2}\right) \wedge\right. \\
N-2 \\
\left.\left.{ }_{G}^{N-1}{ }^{N-1}\right)\right)
\end{gathered}
$$

where

$\mu_{G} N-1\left(x_{N-1}\right)=\operatorname{Max}_{N-1}\left(\mu_{N-1}\left(u_{N-1}\right) \wedge \mu_{G} N\left(f\left(x_{N-1}, u_{N-1}\right)\right)\right)$

may be regarded as the membership function of a fuzzy goal at time $t=$ $\mathrm{N}-1$ which is induced by the given goal $\mathrm{G}^{\mathrm{N}}$ at time $t=N$.

On repeating this backward iteration, which is a simple instance of dynamic programming, we obtain the set of recurrence equations

$$
\mu_{G} N-\nu\left(x_{N-\nu}\right)=\operatorname{Max}_{u_{N}-\nu}\left(\mu\left(u_{N-\nu}\right) \wedge \mu_{G}{ }^{N-v+1}\left(x_{N-v+1}\right)\right)
$$

$$
x_{N-v+1}=f\left(x_{N-v}, u_{N-v}\right), \quad v=1, \ldots, N,
$$

which yield the solution to the problem. Thus, the maximizing decision $u_{0}^{M}, \ldots, u_{N-1}^{M}$, is given by the successive maximizing values of $u_{N-v}$ in (31), with $u_{N-v}^{M}$ defined as a function of $x_{N-v}, v=1, \ldots, N$. 
Example. As a simple illustration, consider a system with three states $\sigma_{1}, \sigma_{2}, \sigma_{3}$ and two Inputs $\alpha_{1}$ and $\alpha_{2}$. Assume $N=2$ for simplicity. Let the fuzzy goal at time $t=2$ be defined by a membership function $\mu_{G}{ }^{2}$ whose values are given by

$\mu_{G}{ }^{2}\left(\sigma_{1}\right)=0.3 ; \mu_{G}{ }^{\left(\sigma_{2}\right)}=1 ; \mu_{G}{ }^{2}\left(\sigma_{3}\right)=0.8$.

Furthermore, let the fuzzy constraints at $t=0$ and $t=1$ be defined respectively by

$\mu_{0}\left(\alpha_{1}\right)=0.7, \mu_{0}\left(\alpha_{2}\right)=1 ; \mu_{1}\left(\alpha_{1}\right)=1, \mu_{1}\left(\alpha_{2}\right)=0.6$.

The state transition table which defines the function $f$ in (25) is assumed to be

\begin{tabular}{c|ccc}
$u_{t} x_{t}$ & $\sigma_{1}$ & $\sigma_{2}$ & $\sigma_{3}$ \\
\hline$\alpha_{1}$ & $\sigma_{1}$ & $\sigma_{3}$ & $\sigma_{1}$ \\
$\alpha_{2}$ & $\sigma_{2}$ & $\sigma_{1}$ & $\sigma_{3}$
\end{tabular}

Using (30), the membership function of the fuzzy goal induced at $t=1$ is found to be

$$
\mu_{G}{ }^{1}\left(\sigma_{1}\right)=0.6 ; \mu_{G}{ }^{1}\left(\sigma_{2}\right)=0.8 ; \mu_{G}{ }^{1}\left(\sigma_{3}\right)=0.6
$$


and the corresponding maximizing decision 1a given by

$$
\begin{aligned}
& \pi_{1}\left(\sigma_{1}\right)=\alpha_{2} ; \pi_{1}\left(\sigma_{2}\right)=\alpha_{1} ; \pi_{1}\left(\sigma_{3}\right)=\alpha_{2} \\
& \text { S1milar1y, for } t=0 \\
& \mu_{G} \circ\left(\sigma_{1}\right)=0.8 ; \mu_{G} \circ\left(\sigma_{2}\right)=0.8 ; \mu_{G} \circ\left(\sigma_{3}\right)=0.6
\end{aligned}
$$

and

$\pi_{a}\left(\sigma_{1}\right)=\alpha_{2} ; \pi_{o}\left(\sigma_{2}\right)=\alpha_{1}$ or $\alpha_{2} ; \pi_{o}\left(\sigma_{3}\right)=\alpha_{1}$ or $\alpha_{2}$.

Thus, If the initial state (at $t=0$ ) is $\sigma_{1}$, then the maximizing deciston is $\alpha_{2}, \alpha_{1}$ and the corresponding value of $\mu_{G}{ }^{2}$ is 0.8 .

Next, we turn to a more general multistage decision process in which the system under control is stochastic, while the goal and the constraints are fuzzy.

\section{Stochastic Systems in a Fuzzy Environment}

As in the proceding problem, assume that the termination time $N$ is fixed and that an Intial state $x_{0}$ is specified. The system Is assumed to be characterized by a conditional probability function $p\left(x_{t+1} \mid x_{t}, u_{t}\right)$. The problem is to maximize the probability of attainment 
of the fuzzy goal at time $N$, subject to the fuzzy constraints $c^{0}, \ldots$, $\mathrm{c}^{\mathrm{N}-1}$.

If the fuzzy goal $\mathrm{G}^{\mathrm{N}}$ is regarded as a fuzzy event ${ }^{10}$ in $\mathrm{X}$, then the conditional probability of this event given $x_{\mathrm{N}-1}$ and $u_{\mathrm{N}-1}$ is expressed by

$$
\begin{aligned}
\operatorname{Prob}\left(G_{N} \mid x_{N-1}, u_{N-1}\right) & =E \mu_{G^{N}}\left(x_{N}\right) \\
& =\sum_{x_{N}} p\left(x_{N} \mid x_{N-1}, u_{N-1}\right) \mu_{G}\left(x_{N}\right)
\end{aligned}
$$

where $E$ denotes the conditional expectation and $\mu_{G^{N}}$ is the membership function of the given fuzzy goal.

We observe that (32) expresses Prob ( $\left.G_{N} \mid x_{N-1}, u_{N-1}\right)$ or, equivalently, $E \mu_{G}\left(x_{N}\right)$, as a function of $x_{N-1}$ and $u_{N-1}$, just as in the preceding problem $\mu_{G}{ }_{N}\left(x_{N}\right)$ was expressed as a function of $x_{N-1}$ and $u_{N-1}$ via (25). This implies that $E \mu_{G^{N}}\left(x_{N}\right)$ can be treated in the same way as $\mu_{G}{ }_{\mathrm{N}}\left(\mathrm{x}_{\mathrm{N}}\right)$ was treated in the non-stochastic case, thus making it possible to reduce the solution of the problem under consideration to that of the preceding problem.

More specifically, the recurrence equations (31) are replaced by 
$\mu_{G}{ }^{N-v}\left(x_{N-v}\right)=\operatorname{Max}_{u_{N-v}}\left(\mu_{N-v}\left(u_{N-v}\right), \quad E \mu_{G N-v+1}\left(x_{N-v+1}\right)\right)$

$E \mu_{G}{ }_{N-v+1}\left(x_{N-v+1}\right)=\sum_{x_{N-v+1}} p\left(x_{N-v+1} \mid x_{N-v}, u_{N-v}\right) \mu_{G}^{N-v+1}{ }\left(x_{N-v+1}\right)$

where, as before, ${ }_{G^{N}-\nu}\left(x_{N-\nu}\right)$ denotes the membership function of the fuzzy goal at $t=N-v$ induced by the fuzzy goal at $t=N-v+1$, $\nu=1, \ldots, N$. These equations yield a solution to the problem, as is 11lustrated by the following example.

Example. As in the preceding example, we assume that the system has three states $\sigma_{1}, \sigma_{2}, \sigma_{3}$ and two inputs $\alpha_{1}, \alpha_{2}, \mathrm{~N}$ is assumed to be equal to 2 , and the probability function $p\left(x_{t+1} \mid x_{t}, u_{t}\right)$ is given by the following two tables, corresponding to $u_{t}=\alpha_{1}$, and $u_{t}=\alpha_{2}$, respectively.

I.

$$
u_{t}=\alpha_{1}
$$

\begin{tabular}{c|ccc}
$\mathbf{x}_{t}^{\mathbf{x}_{t+1}}$ & $\sigma_{1}$ & $\sigma_{2}$ & $\sigma_{3}$ \\
\hline$\sigma_{1}$ & 0.8 & 0.1 & 0.1 \\
$\sigma_{2}$ & 0 & 0.1 & 0.9 \\
$\sigma_{3}$ & 0.8 & 0.1 & 0.1
\end{tabular}

II. $\quad u_{t}=\alpha_{2}$

\begin{tabular}{c|ccc}
$\mathbf{x}_{t} \mathrm{x}_{\mathrm{t}+1}$ & $\sigma_{1}$ & $\sigma_{2}$ & $\sigma_{3}$ \\
\hline$\sigma_{2}$ & 0.1 & 0.9 & 0 \\
$\sigma_{3}$ & 0.8 & 0.1 & 0.1 \\
$\sigma_{3}$ & 0.1 & 0 & 0.9
\end{tabular}

The entries in these tables are the values of $p\left(x_{t+1} \mid x_{t}, u_{t}\right)$. 
Thus, the entry 0.8 in the position $\left(\sigma_{1}, \sigma_{2}\right)$ in the first table signifies that if the system is in state $\sigma_{1}$ at time $t$ and input $\alpha_{1}$ is applied, then with probability 0.8 the state at time $t+1$ will be $\sigma_{2}$.

The fuzzy goal at $t=2$ is assumed to be the same as in the preceding example, that is

$$
\mu_{G}{ }^{2}\left(\sigma_{1}\right)=0.3 ; \mu_{G}{ }^{2}\left(\sigma_{2}\right)=1 ; \mu_{G}{ }^{2}\left(\sigma_{3}\right)=0.8
$$

Likewise, the constraints are assumed to be the same. Thus

$$
\mu_{0}\left(\alpha_{1}\right)=0.7, \mu_{0}\left(\alpha_{2}\right)=1 ; \mu_{1}\left(\alpha_{1}\right)=1, \mu_{1}\left(\alpha_{2}\right)=0.6
$$

$$
\text { Using (33), we compute } E \mu_{G}{ }^{2}\left(x_{2}\right) \text { as a function of } x_{1} \text { and }
$$

$u_{1}$. Tabulating the results, we have

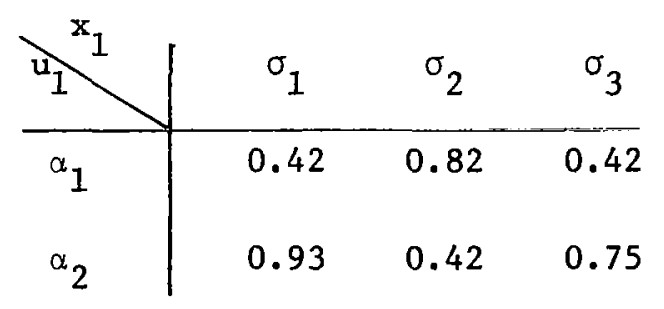

$$
\text { Next, using (33) with } \nu=1 \text { and computing } \mu_{G}{ }_{1}\left(x_{1}\right) \text { we }
$$

obtain

$$
\mu_{G}{ }^{1}\left(\sigma_{1}\right)=0.6 ; \mu_{G}{ }^{1}\left(\sigma_{2}\right)=0.82 ; \mu_{G}{ }\left(\sigma_{3}\right)=0.6
$$


which correspond to the following values of the maximal policy function

$$
\pi_{1}\left(\sigma_{1}\right)=\alpha_{2} \quad ; \quad \pi_{1}\left(\sigma_{2}\right)=\alpha_{1} ; \quad \pi_{1}\left(\sigma_{3}\right)=\alpha_{2} ;
$$

The final iteration with $\nu=2$ ylelds

$$
\begin{aligned}
& \begin{array}{c|ccc}
\pi_{0}^{x_{0}} & \sigma_{1} & \sigma_{2} & \sigma_{3} \\
\hline \alpha_{1} & 0.62 & 0.62 & 0.62 \\
\alpha_{2} & 0.8 & 0.62 & 0.60
\end{array} \\
& \mu_{G}{ }^{\circ}\left(\sigma_{1}\right)=0.8 ; \mu_{G}{ }^{\circ}\left(\sigma_{2}\right)=0.62 ; \mu_{G}{ }^{\circ}\left(\sigma_{3}\right)=0.62 \\
& \pi_{0}\left(\sigma_{1}\right)=\alpha_{1} ; \pi_{0}\left(\sigma_{2}\right)=\alpha_{1} \text { or } \alpha_{2} ; \pi_{0}\left(\sigma_{3}\right)=\alpha_{1} \text {. }
\end{aligned}
$$

The values of $\mu_{G^{\circ}}$ in (61) represent the probabilities of attaining the given goal at $t=2$ starting with $\sigma_{1}, \sigma_{2}$ and $\sigma_{3}$, respectively, assuming that the inputs are determined by the maximal policy function $\pi_{t}$, that is, $u_{t}=\pi_{t}\left(x_{t}\right) \quad\left(t=0,1, x_{t}=\sigma_{1}, \sigma_{2}, \sigma_{3}, u_{t}=\right.$ $\alpha_{1}, \alpha_{2}$ ) whose values are given in (60) and (62).

Comment. It should be noted that when the fuzzy goal at time $\mathrm{N}$ is defined in such a way that the probability of attaining it is small for all values of $x_{N-1}$ and $u_{N-1}$, it may be necessary to normallze the 
fuzzy goal induced at time $\mathrm{N}-1$ before finding its intersection with $\mathrm{C}_{\mathrm{N}-1}$, for otherwise the decision would be unffluenced by the constraints. To be consistent, such normalization may have to be carried out at each stage of the decision process. Although we shall not dwe1l further upon this aspect of the problem in the present paper, it should be emphasized that it is by no means a trivial one and requires a more thorough analysis.

\section{Systems with Implicitly Defined Termination Time}

In the preceding cases, we have assumed that the termination time, $N$, is fixed a priori. In the more general case which we shall consider in this section, the termination time is assumed to be determined implicitly by a subsidiary condition of the form $x_{N} \in T$, where $T$ is a specified non-fuzzy subset of $X$ termed the termination set. Thus, the process terminates when the state of the system under control enters, for the first time, a specified subset of the state space. In this case, the goal is defined as a fuzzy set $G$ in $T$, rather than in $X$.

More concretely, assume that the system under control, A, is a deterministic system characterized by a state equation of the form

$$
x_{t+1}=f\left(x_{t}, u_{t}\right) \quad, \quad t=0,1,2, \ldots
$$

where $x_{t}$ ranges over $x=\left\{\sigma_{1}, \ldots, \sigma_{\ell}, \sigma_{\ell A 1}, \ldots, \sigma_{n}\right\}$, in which $T=\left\{\sigma_{\ell+1}, \ldots\right.$, $\left.\sigma_{n}\right\}$, constitutes the termination set. As before, $f$ is assumed to be a *In its conventional (non-fuzzy) formulation, this case plays an important role in the theory of optimal control and Markoffian decision processes. Some of the more relevant papers on this subject are cited in the list of references. 



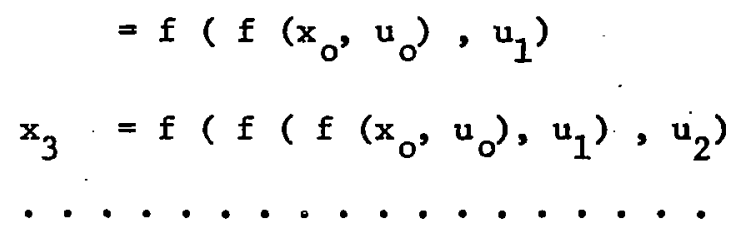

Note that, as in (26), the C's in (35) should be regarded as fuzzy sets in the product space $U \mathrm{xU} \mathrm{x}_{\ldots} \ldots \mathrm{x}$ Ux $\mathrm{T}$. Another point that should be noted is that $D\left(x_{0}\right)$ is uniquely determined by (35) for each $x_{0}$, with the understanding that $D\left(x_{0}\right)$ is empty if there is no finite sequence of inputs $u_{0}, \ldots, u_{N-1}$ which takes the initial state $x_{0}$ into $T$. In this event, we shall say that $T$ is not reachable from the initial state.

From (35), we can readily derive a simpler implicit equation which is satisfied by $D\left(x_{0}\right)$. Specifically, in virtue of the timeinvariance of $\mathrm{A}$ and the time-independence of the goal and constraint sets, (35) implies

$$
D\left(x_{t}\right)=C\left(x_{t}\right) \cap C\left(x_{t+1}\right) \cap \ldots \cap C\left(x_{i+N-1}\right) \cap G
$$

for $t=0,1,2, \ldots$. In particular,

$$
D\left(x_{t+1}\right)=C\left(x_{t+1}\right) \cap \ldots \cap C\left(x_{t+N-1}\right) \cap G
$$

and hence (37) can be written as

$$
D\left(x_{t}\right)=C\left(x_{t}\right) \cap D\left(x_{t+1}\right)
$$


or, using (34),

$$
D\left(x_{t}\right)=C\left(x_{t}\right) \cap D\left(f\left(x_{t}, u_{t}\right)\right) \quad, \quad t=0,1,2, \ldots
$$

which is the desired implicit equation. Expressed in terms of the membership functions of the sets in question, this equation assumes the following form (for $t=0$ )

$\mu_{D}\left(u_{0}, \ldots, u_{N-1} \mid x_{0}\right)=\mu_{C}\left(u_{0} \mid x_{0}\right) \wedge \mu_{D}\left(u_{1}, \ldots, u_{N-1} \mid f\left(x_{0}, u_{0}\right)\right)$

where the termination time $N$ is also a function of $x_{0}$ and $u_{0}, u_{1}, u_{2}, \ldots$ through the state equation (34) and the termination condition $x_{N} \in T$, with $x_{\mathrm{O}} \notin \mathrm{T}, \ldots, \mathrm{x}_{\mathrm{N}-\mathrm{I}} \notin \mathrm{T}$.

Now suppose that the successive inputs $u_{0}, u_{1}, \ldots, u_{N-1}$ are determined by a stationary (time-invariant) policy function $\pi, \pi$ : $T^{\prime} \rightarrow V$, which associates with each state $x_{t}$ in $T^{\prime}$ an input $u_{t}$ which should be applied to $A$ when it is in state $x_{t}$. Thus,

$$
u_{t}=\pi\left(x_{t}\right), t=0, \ldots, N-1, x_{t} \varepsilon T^{\prime}
$$

Since $u_{0}, \ldots, u_{N-1}$ are determined by $x_{0}$ and $\pi$ through (42) and the state equation (34), the membership function of $D\left(x_{0}\right)$ can be written as $\mu_{D}\left(x_{0} \mid \pi\right)$. Similarly, $\mu_{c}\left(u_{0} \mid x_{0}\right)$ can be written as $\mu_{C}\left(\pi\left(x_{0}\right) \mid x_{0}\right)$, and $\mu_{D}\left(u_{1}, \ldots, u_{N-1} \mid f\left(x_{0}, u_{0}\right)\right)$ as $\mu_{D}\left(f\left(x_{0}, \pi\left(x_{0}\right)\right) \mid \pi\right)$. 
With these substitutions, (41) assumes the more compact form

$$
\mu_{D}\left(x_{0} \mid \pi\right)=\mu_{C}\left(\pi\left(x_{0}\right) \mid x_{0}\right) \mu_{D}\left(f\left(x_{0}, \pi\left(x_{0}\right)\right) \mid \pi\right), x_{0} \varepsilon T^{\prime},
$$

which in effect is a system of $\ell$ equations (one for each value of $x_{0}$ ) in the $\mu_{D}$. This system of equations determines $\mu_{D}$ as a function of $x_{0}$ for each $\pi$, with the understanding that $\mu_{D}=0$ if under $\pi$ the process does not terminate, that 1 , there does not exist a finite $N$ such that $\mathrm{x}_{\mathrm{N}} \varepsilon \mathrm{T}$. Furthermore, it is understood that $\mu_{\mathrm{D}}=\mu_{\mathrm{G}}$ for states in $\mathrm{T}$.

It is easy to demonstrate that (43) has a unique solution. Specifically, by decomposing the set of states $T^{\prime}=\left\{\sigma_{1}, \ldots, a_{\ell}\right\}$ into disjoint subsets $T_{1}^{\prime}, \ldots, T_{K}^{\prime}$, where $T_{\lambda}^{\prime}, \lambda=1, \ldots, K$, represents the set of states from which $\mathrm{T}$ is reachable in $\lambda$ steps, 1t is read1ly seen that the equations in (43) corresponding to the $x_{0}$ which are in $T_{1}$ yield uniquely the respective values of $\mu_{D}$. In terms of these, the equations in (43) corresponding to the $x_{0}$ in $T_{2}$ yield uniquely the values of $\mu_{D}$ for $x_{0}$ in $T_{2}$. Continuing in this manner, all the $\mu_{D}$ 's can be determined uniquely by successively solving subsets of the system of equations (43) for the blocks of variables in $\mathrm{T}_{1}^{\prime}, \ldots, \mathrm{T}_{K}^{\prime}$.

For our purposes, it will be convenient to represent a policy $\pi$ as a policy vector

$$
\pi=\left(\pi\left(\sigma_{1}\right), \ldots, \pi\left(\sigma_{\ell}\right)\right)
$$


whose $1^{\text {th }}$ component $1=1, \ldots, l$, Is the input which must be applied when $A$ is in state $\sigma_{1}$. Note that $\pi\left(\sigma_{1}\right)$ ranges over the set $U=\left\{\alpha_{1}, \ldots\right.$, $\left.\alpha_{m}\right\}$ and thus that there are $m^{l}$ distinct policies in the policy space.

With reference to the system of equations (43), let

$$
\mu_{D}(\pi)=\left(\mu_{D}\left(\sigma_{1} \mid \pi\right), \ldots, \mu_{D}\left(\sigma_{n} \mid \pi\right)\right)
$$

be an $n$-vector, termed the goal attainment vector, whose components are the values of the membership function of $D$ at $\sigma_{1}, \ldots, \sigma_{n}$ (corresponding to policy $\pi$ ). It is natural to define a partial ordering in the policy space by the inequality

$$
\pi^{\prime} \geq \pi^{\prime \prime} \Rightarrow \mu_{D}\left(\pi^{\prime}\right) \geq \mu_{D}\left(\pi^{\prime \prime}\right)
$$

which means that a policy $\pi^{\prime}$ is better than or equal to a policy $\pi^{\prime \prime}$ if and only if $\mu_{D}\left(\sigma_{i} \mid \pi^{\prime}\right) \geq \mu_{D}\left(\sigma_{1} \mid \pi^{\prime \prime}\right)$ for $i=1, \ldots, n$. Then, a policy $\pi$ will be sald to be maxlmal if and only if $\pi$ is better than or equal to every policy in the policy space.

Does there exist a maximal policy for the problem under consideration? The answer to this question is in the affirmative. This assertion can be proved rigorously,* but it will suffice for our purposes

* A proof for the case of a stochastic finite-state system is given in Ref. 13 . 
to regard it as a consequence of the alternation principle ${ }^{14}$ - a principle of broad validity which in concrete cases can be asserted as a provable theorem.

Specifically, let $\pi^{\prime}$ and $\pi^{\prime \prime}$ be two arbitrary policy vectors, with $\mu_{D}\left(\pi^{\prime}\right)$ and $\mu_{D}\left(\pi^{\prime \prime}\right)$ being the corresponding goal attainment vectors. Using $\pi^{\prime}$ and $\pi^{\prime \prime}$, let us construct a policy vector $\pi$ in accordance with the following rules:

$$
\begin{aligned}
\pi_{i} & =\pi_{i}^{\prime} \text { if } \mu_{D}\left(\sigma_{i} \mid \pi^{\prime}\right) \geq \mu_{D}\left(\sigma_{i} \mid \pi^{\prime \prime}\right) \\
& =\pi_{i}^{\prime \prime} \text { if } \mu_{D}\left(\sigma_{i} \mid \pi^{\prime}\right)<\mu_{D}\left(\sigma_{i} \mid \pi^{\prime \prime}\right)
\end{aligned}
$$

for each component $\pi_{i}$ of $\pi, i=1, \ldots, \&$. Then, according to the alternation principle, $\pi \geq \pi^{\prime}$ and $\pi \geq \pi^{\prime \prime}$, that is, $\pi$ is better than or equal to both $\pi^{\prime}$ and $\pi^{\prime \prime}$. From this and the finiteness of the policy space it follows at once that there exists a maximal policy.

From (43) it is a simple matter to derive a functional equation satisfied by the goal attainment vector corresponding to the maximal policy. Thus, let

$$
\mu_{D}^{M}=\underset{\pi}{\operatorname{Max}} \mu_{D}(\pi)
$$

and let $P(\pi)$ be an $n \times n$ matrix of zeros and ones whose $i j$ th element is 
one if and only if $\sigma_{j}=f\left(\sigma_{i}, \pi\left(\sigma_{i}\right)\right)$, that is, the state $\sigma_{j}$ is the immediate successor of $\sigma_{i}$ under policy $\pi$.

Furthermore, let $\mu_{C}(\pi)$ denote a vector whose 1 th component is $\mu_{C}\left(\pi\left(\sigma_{1}\right) \mid \sigma_{1}\right)$. Then, on taking the maximum of both sides of (43), we obtain

$$
\mu_{D}^{M}=\underset{\pi}{\operatorname{Max}}\left(\mu_{C}(\pi) \wedge P(\pi) \mu_{D}^{M}\right)
$$

which is the desired functional equation for $\mu_{D}^{M}$. Although different in detail, equation (49) is of the same general form as the functional equations arising in the theory of Markoffian decision processes. $12-27$ Its solution, however, is considerably simpler to obtain because of the distributivity of $\operatorname{Max}$ and $\Lambda$.

Specifically, let $\pi^{1}, \ldots, \pi^{r}$, where $r=m^{\ell}$, denote the $\mathrm{m}^{\ell}$ distinct policy vectors. Then, on using $\vee$ in place of Max, (49) becomes

$\mu_{D}^{M}=\left(\mu_{C}\left(\pi^{\prime}\right) \wedge P\left(\pi^{\prime}\right) \mu_{D}^{M}\right) \vee \ldots v\left(\mu\left(\pi^{r}\right) \wedge P\left(\pi^{r}\right) \mu_{D}^{M}\right)$

Taking advantage of the distributivity of $v$ and $\Lambda$, and factoring like terms, we can put (50) into a much simpler form which, written as a system of equations in the components of $\mu_{D}^{M}$, reads 
$\mu_{D}^{M}\left(\sigma_{i}\right)=v_{j}^{v}\left(\mu_{C}\left(\alpha_{j} \mid \sigma_{i}\right) \wedge \mu_{D}^{M}\left(f\left(\sigma_{i}, \alpha_{j}\right)\right)\right), \quad \begin{aligned} & 1=1, \ldots, n \\ & j=1, \ldots, m\end{aligned}$

where $\alpha_{j}=\pi\left(\sigma_{1}\right)=$ input under policy $\pi$ in state $\sigma_{1} ; \mu_{D}^{M}\left(\sigma_{1}\right)=1$ th component of the maximal goal attainment vector; $f\left(\sigma_{i}, \alpha_{j}\right)=$ successor state* of $\sigma_{i}$ for input $\alpha_{j}$, with $f\left(\sigma_{i}, \alpha_{j}\right)=\sigma_{i}$ for $1=1 l+1, \ldots, n$ (that is, for $\sigma_{i}$ in the termination set $\left.T\right)$; $\mu_{C}\left(\alpha_{j} \mid \sigma_{i}\right)=$ value of the membership function of the constraint $C$ in state $\sigma_{i}$ for input $\alpha_{j}$, with $\mu_{c}\left(\alpha_{j} \mid \sigma_{i}\right)=1$ for $i=\ell+1, \ldots, n ;$ and for $i=\ell+1, \ldots, n, \mu_{D}^{M}\left(\sigma_{i}\right)=\mu_{G}\left(\sigma_{i}\right)=$ value of the membership function of the given goal $G$ at $\sigma_{i}$. Thus, the $\mu_{D}^{M}\left(\sigma_{1}\right), i=1, \ldots, l$, are the unknowns in $(51)$, while the $\mu_{D}^{M}\left(\sigma_{i}\right), i=$ $\ell+1, \ldots, n$, and the $\mu_{C}\left(\alpha_{j} \mid \sigma_{i}\right), i=1, \ldots, n, j=1, \ldots, m$, are given constants.

To make the solution of (51) more transparent, it is helpful to simplify the notation in (51) by leting the unknowns in (51) be denoted by $\omega_{i}$, that is, $\omega_{i}=\mu_{D}^{M}\left(\sigma_{i}\right)$ for $1=1, \ldots, \ell$. Furthermore, let the product and plus symbols denote $\Lambda$ and $V$, respectively. Then, (51) can be written more compactly in matrix form as

$$
\omega=B \omega+\gamma
$$

where $\omega=\left(\omega_{1}, \ldots, \omega_{l}\right), \gamma=\left(\gamma_{1}, \ldots, \gamma_{l}\right), B=\left(b_{i k}\right)$; furthermore,

* Note that the successor states in (49) are defined by $P(\pi)$. 


$$
\begin{aligned}
b_{1 k}= & 0 \text { if } \sigma_{k} \text { is not an immediate successor of } \sigma_{1} ; \\
b_{1 k}= & v \mu_{c}\left(\alpha_{p} \mid \sigma_{1}\right), \text { where the } \alpha_{p} \text { are inputs which take } \sigma_{1} \text { into } \sigma_{k} ;
\end{aligned}
$$

and

$$
\gamma_{i}=\underset{j}{V}\left(\mu_{c}\left(\alpha_{j} \mid \sigma_{i}\right) \wedge \mu_{G}\left(f\left(\sigma_{i}, \alpha_{j}\right)\right)\right)
$$

with the understanding that $\mu_{G}\left(\dot{\sigma}_{i}\right)=0$ for states outside the termination set $T$.

Having put (51) into the form of a linear equation (52), it is easy to show that (53) and hence (51) can be solved by iteration. Specifically, let $\omega^{\circ}=(0, \ldots, 0)$ and

$$
\omega^{s+1}=B \omega^{s}+\gamma \quad, \quad s=0,1,2, \ldots
$$

Then, by induction, the sequence $\omega^{0}, \omega^{1}, \omega^{2}, \ldots$ is monotone non-decreasing. For, assume that $\omega^{k+1} \geq \omega^{k}$ for some $k$. Using (54), we have

$$
\omega^{k+2}=B \omega^{k+1}+\gamma \geq B \omega^{k}+\gamma=\omega^{k+1} \text {, }
$$

and noting that $\omega^{1} \geq \omega^{\alpha}=0$, it follows that $\omega^{s+1} \geq \omega^{s}$ for $s=0,1,2, \ldots$.

$$
\text { Since the sequence } \omega^{0}, \omega^{1}, \ldots \text { is monotone non-decreasing }
$$
and bounded from above by $\omega=(1, \ldots, 1)$, it follows that it converges to 
the solution of (52), that is, to the first $l$ components* of the maximal goal attainment vector $\mu_{D}^{M}$. Actually, a more detalled argument shows that (54) yields the solution of (52) in not more than $l$ iterations. In essence, this follows from the fact that if $T$ is reachable from a state in $T^{\prime}$, then $1 t$ is reachable in $\ell$ or fewer steps.

To gain an intuitive insight into the above solution, it is helpful to interpret the transition from (49) to (51) with the aid of the state diagram of A. Thus, for concreteness assume that A has five states, with transitions corresponding to various inputs shown in Fig.2. In this diagram, the number associated with the branch leading from $\sigma_{i}$ to its successor state via input $\alpha_{j}$ is the value of $\mu_{c}\left(\alpha_{j} \mid \sigma_{i}\right)$. States $\sigma_{4}$ and $\sigma_{6}$ are in the termination set and the corresponding values of $\mu_{G}\left(\sigma_{i}\right)$ are shown alongside. The indicated values of the $\mu_{c}\left(\alpha_{j} \mid \sigma_{i}\right)$ correspond to the constraint sets

$$
\begin{aligned}
& C\left(\sigma_{1}\right)=\left\{\left(\alpha_{1}, 0.6\right),\left(\alpha_{2}, 1\right)\right\} \\
& C\left(\sigma_{2}\right)=\left\{\left(\alpha_{1}, 0.8\right),\left(\alpha_{2}, 1\right)\right\} \\
& C\left(\sigma_{3}\right)=\left\{\left(\alpha_{1}, 1\right),\left(\alpha_{2}, 0.7\right)\right\}
\end{aligned}
$$

For the system in question, the state transition function $f\left(\sigma_{i}, \alpha_{j}\right)$ is given by the following table * The remaining $\mathrm{n}-\ell$ components of $\mu_{\mathrm{D}}^{\mathrm{M}}$ are given by the corresponding
components of $\mu_{\mathrm{G}}$. 


\begin{tabular}{c|ccccc}
$\alpha_{j}$ & $\sigma_{1}$ & $\sigma_{2}$ & $\sigma_{3}$ & $\sigma_{4}$ & $\sigma_{5}$ \\
\hline$\alpha_{1}$ & $\sigma_{4}$ & $\sigma_{3}$ & $\sigma_{5}$ & $\sigma_{4}$ & $\sigma_{5}$ \\
$\alpha_{2}$ & $\sigma_{2}$ & $\sigma_{2}$ & $\sigma_{1}$ & $\sigma_{4}$ & $\sigma_{5}$
\end{tabular}

From this table, it is easy to construct the matrix $P(\pi)$ for any given policy. For example, for $\pi=\left(\alpha_{2}, \alpha_{1}, \alpha_{2}\right)$, we have

$$
P\left(\alpha_{2}, \alpha_{1}, \alpha_{2}\right)=\left[\begin{array}{lllll}
0 & 1 & 0 & 0 & 0 \\
0 & 0 & 1 & 0 & 0 \\
1 & 0 & 0 & 0 & 0 \\
0 & 0 & 0 & 1 & 0 \\
0 & 0 & 0 & 0 & 1
\end{array}\right]
$$

The system of equations (51) is obtained by reversing the direction of flow in each branch (see Fig.3) and treating the states in $\mathrm{T}$, that is, $\sigma_{4}$ and $\sigma_{5}$ as sources, with the states in $T^{\prime}$, that is, $\sigma_{1}$, $\sigma_{2}$ and $\sigma_{3}$, playing the role of receptors (sinks). From the diagram shown in Fig.3, the equations in (51) can be written by inspection. Thus,

$$
\begin{aligned}
& \mu_{\mathrm{D}}^{\mathrm{M}} \quad\left(\sigma_{1}\right)=\left(0.6 \wedge \mu_{\mathrm{D}}^{\mathrm{M}}\left(\sigma_{4}\right)\right) \vee\left(1 \wedge \mu_{\mathrm{D}}^{\mathrm{M}}\left(\sigma_{2}\right)\right) \\
& \mu_{\mathrm{D}}^{\mathrm{M}} \quad\left(\sigma_{2}\right)=\left(0.8 \wedge \mu_{\mathrm{D}}^{\mathrm{M}}\left(\sigma_{3}\right)\right) \vee\left(1 \wedge \mu_{\mathrm{D}}^{\mathrm{M}}\left(\sigma_{2}\right)\right) \\
& \mu_{\mathrm{D}}^{\mathrm{M}} \quad\left(\sigma_{3}\right)=\left(1 \wedge \mu_{\mathrm{D}}^{\mathrm{M}}\left(\sigma_{5}\right)\right) \vee\left(0.7 \wedge \mu_{\mathrm{D}}^{\mathrm{M}}\left(\sigma_{1}\right)\right) \\
& \mu_{\mathrm{D}}^{\mathrm{M}} \quad\left(\sigma_{4}\right)=\mu_{\mathrm{G}}\left(\sigma_{4}\right)=1 \\
& \mu_{\mathrm{D}}^{\mathrm{M}} \quad\left(\sigma_{5}\right)=\mu_{G}\left(\sigma_{5}\right)=0.8
\end{aligned}
$$


Employing the simplified notation in which $\Lambda$ and $v$ are replaced by the product and sum, respectively, and $\omega_{1}=\mu_{D}^{M}\left(\sigma_{1}\right), 1=1$, 2,3 , the system of equations (56) becomes

$$
\omega=B \omega+\gamma
$$

where

$$
B=\left[\begin{array}{lll}
0 & 1 & 0 \\
0 & 1 & 0.8 \\
0.7 & 0 & 0
\end{array}\right] \quad \gamma=\left[\begin{array}{l}
0.6 \\
0 \\
0.8
\end{array}\right]
$$

$$
\text { Letting } \omega^{\circ}=(0,0,0) \text {, we obtain on first interation } \omega^{1}=
$$
$(0.6,0,0.8)$

Subsequent iterations yield

$$
\begin{aligned}
& \omega^{2}=(0.6,0.8,0.8) \\
& \omega^{3}=(0.8,0.8,0.8) \\
& \omega^{4}=(0.8,0.8,0.8)
\end{aligned}
$$

Thus, $w^{3}=(0.8,0.8,0.8)$ is the solution of $(57)$.

To visualize the iteration process, imagine that each of the sources in Fig.3 (which are the absorbing states in Fig.2) generates balls of various diameters, with $\sigma_{i}, i=\ell+1, \ldots, n$, generating balls 
of diameters ranging from 0 to $\mu_{G}\left(\sigma_{i}\right)$. Furthermore, Imagine that a branch in Fig. 2 which leaves state $\sigma_{1}$ via input $\alpha_{f}$, is a pipe of diameter $\mu_{C}\left(\alpha_{j} \mid \sigma_{1}\right)$ which can carry balls of diameter $\leq \mu_{C}\left(\alpha_{j} \mid \sigma_{f}\right)$ along the reverse direction, that is, along the direction shown in Fig.3. Thus, the diagram of Fig. 3 may be visualized as a network of pipes whose diameters are Indicated in the diagram and which can carry balls of lesser or equal diameter in the indicated directions. The states in the termination set $\left(\sigma_{4}\right.$ and $\left.\sigma_{5}\right)$ play the role of sources of balls of diameters up to $\mu_{G}\left(\sigma_{4}\right)$ and $\mu_{G}\left(\sigma_{5}\right)$ respectively, while the remaining states $\left(\sigma_{1}, \sigma_{2}\right.$ and $\sigma_{3}$ ) act as receptors. Because the absorbing states act as sources, we shall refer to the method of solution described above as a reverseflow technique.

Now assume that it takes one unit of time for the balls to travel from a node of the network of Fig. 3 to another node. If we start with no balls at $\sigma_{1}, \sigma_{2}$ and $\sigma_{3}$ at time 0 , then at time $t=1$ the maximum diameters of balls at $\sigma_{1}, \sigma_{2}$ and $\sigma_{3}$ will be, respectively, $\omega_{1}^{1}, \quad \omega_{2}^{1}$ and $\omega_{3}^{1}$, where $\omega^{1}=\left(\omega_{1}^{1}, \omega_{2}^{1}, \omega_{3}^{1}\right)$ is the first iterate of (57). At time $t=2$, the maximum diameters of balls will be given by $\omega^{2}$ and at time $t=3$ by $\omega^{3}$. Since it takes no more than three units of time for any ball to travel from its source to any node in the network, there will be no further increase in the size of balls at each source upon further iteration. Thus, $\omega^{3}$ gives the maximum diameter of balls at each receptor node and hence is the desired solution of (57). 
Turning to the illustration of (43) and the alternation principle, consider the policy vector $\pi=\left(\alpha_{1}, \alpha_{1}, \alpha_{1}\right)$. For this $\pi$, the system of equations (43) becomes

$$
\begin{aligned}
& \mu_{D}\left(\sigma_{1} \mid \pi\right)=0.6 \wedge \mu_{D}\left(\sigma_{4} \mid \pi\right) \\
& \mu_{D}\left(\sigma_{2} \mid \pi\right)=0.8 \wedge \mu_{D}\left(\sigma_{3} \mid \pi\right) \\
& \mu_{D}\left(\sigma_{3} \mid \pi\right)=1 \wedge \mu_{D}\left(\sigma_{5} \mid \pi\right)
\end{aligned}
$$

In this case, $\sigma_{1}$ and $\sigma_{3}$ are in $T_{1}^{\prime}$ and $\sigma_{2}$ is in $T_{2}^{\prime}$. Noting that $\mu_{D}\left(\sigma_{4} \mid \pi\right)=\mu_{G}\left(\sigma_{4}\right)=1$ and $\mu_{D}\left(\sigma_{5} \mid \pi\right)=\mu_{G}\left(\sigma_{5}\right)=0.8$, we find at once $\mu_{D}\left(\sigma_{1} \mid \pi\right)=0.6 ; \mu_{D}\left(\sigma_{2} \mid \pi\right)=0.8 ; \mu_{D}\left(\sigma_{3} \mid \pi\right)=0.8$ which is the desired solution.

Carrying out the same computation for other policy vectors, we obtain the results tabulated below

\begin{tabular}{c|ccc}
$\pi$ & $\sigma_{1}$ & $\sigma_{2}$ & $\sigma_{3}$ \\
\hline$\left(\alpha_{1}, \alpha_{1}, \alpha_{1}\right)$ & 0.6 & 0.8 & 0.8 \\
$\left(\alpha_{1}, \alpha_{1}, \alpha_{2}\right)$ & 0.6 & 0.6 & 0.6 \\
$\left(\alpha_{1}, \alpha_{2}, \alpha_{1}\right)$ & 0.6 & 0 & 0.8 \\
$\left(\alpha_{1}, \alpha_{2}, \alpha_{2}\right)$ & 0.6 & 0 & 0.6 \\
$\left(\alpha_{2}, \alpha_{1}, \alpha_{1}\right)$ & 0.8 & 0.8 & 0.8 \\
$\left(\alpha_{2}, \alpha_{1}, \alpha_{2}\right)$ & 0 & 0 & 0 \\
$\left(\alpha_{2}, \alpha_{2}, \alpha_{1}\right)$ & 0 & 0 & 0.8 \\
$\left(\alpha_{2}, \alpha_{2}, \alpha_{2}\right)$ & 0 & 0 & 0
\end{tabular}


As a check on the alternation principle, let us take $\pi^{\prime}=\left(\alpha_{1}, \alpha_{1}, \alpha_{2}\right)$ and $\pi^{\prime \prime}=\left(\alpha_{1}, \alpha_{2}, \alpha_{1}\right)$. Using (47) leads to $\pi=\left(\alpha_{1}, \alpha_{1}, \alpha_{1}\right)$. Note that $\pi \geq \pi^{\prime}$ and $\pi \geq \pi^{\prime \prime}$. From inspection of the table, the maximal policy is seen to be $\left(\alpha_{2}, \alpha_{1}, \alpha_{1}\right)$. which agrees with the result obtained by iteration.

The approach to the solution of problems involving implicitly defined termination time which we have described in this section can be extended to more complex decision processes in a fuzzy environment. In particular, the technique employed for solving the functional equation (49) can readily be extended to fuzzy systems in a fuzzy environment. Furthermore, (43) and (49) can be extended also - as in section 4 - to stochastic finitestate systems. Because of limitations on space, we shall not consider these cases in the present paper.

\section{Concluding Remarks}

The task of developing a general theory of decisionmaking in a fuzzy environment is one of very considerable magnitude and complexity. Thus, the results presented in this paper should be viewed as merely a first attempt at constructing a conceptual framework for such a theory.

There are many facets of the theory of decision-making in a fuzzy environment which require more thorough investigation. Among 
these are the question of execution of fuzzy decisions; the way in which the goals and the constraints must be combined when they are of unequal importance or are interdependent; the control of fuzzy systems and the implementation of fuzzy algorithms; the notion of fuzzy feedback and 1ts effect on decision-making; control of systems in which the fuzzy environment is partially defined by exemplification; and decisionmaking in mixed environments, that is, in environments in which the imprecision stems from both randomess and fuzziness. 


\section{References}

1. L. A. Zadeh, "Fuzzy Sets," Information and Cantrol, val.8, pp.338-353, June, 1965.

2. L. A. Zadeh, "Toward a Theory of Fuzzy Systems," NASA CR-1432, September 1969

3. L. A. Zadeh, "Fuzzy Algorithms," Information and Control, vol.12, pp.99-102, February, 1968

4. 5. 5. L. Chang, "Fuzzy Dynamic Programming and the Decision Making Pracess," Proc. 3d Princeton Conference on Infarmation Sciences and Systems, pp.200-203, 1969.

5. K. 5. Fu and T. J. Li, "On the Behavior of Learning Autamata and its Applications," Tech. Rep. TR-EE 68-20, Purdue University, Lafayette, Indiana, Aug. 1968.

6. J. Goguen, "L-fuzzy Sets," Jour. Math. Anal. and Appl., vol.18, pp.145-174, April, 1967.

7. J. G. Brown, "Fuzzy Sets on Boolean Lattices," Rep. No. 1957, Ballistic Research Laboratories, Aberdeen, Maryland, January, 1969. 
8. R. Beliman, R. Kalaba and L. A. Zadeh, "Abstraction and Pattern Classification," Jaur. Math. Anal. and Appl., vol.13, pp.1-7, Janiary, 1966.

9. W. G. Wee, "On Generalization of Adaptive Algorithms and Application of the Fuzzy Set Concept to Pattern Classification," Technical Repart TR-EE-67-7, Purdue University, Lafayette, Indiana, July,1967.

10. L. A. Zadeh, "Probability Measures of Fuzzy Events," Jaur. Math. Anal. and Appl., vol.10, pp.421-427, August, 1968.

11. C. L. Chang, "Fuzzy Topological Spaces," Jour. Math. Analysis and Appl., val.24, pp.182-190, 1968.

12. H. M. Wagnex, "Principles of Dperations Research," Prentice-Hall, Inc. 1969.

13. J. H. Eaton and L. A. Zadeh, "Optimal Pursuit Strategies in DiscreteState Probabilistic Systems," Jour. Basic Engineering (ASME), vol.84, Series D, pp.23-29, March, 1962.

14. 3. H. Eaton and L. A. Zadeh, "An Alternation Principle for Optimal Control, "Autamation and Remote Contral,vol.24, pp.328-330,March,1963.

15. R. E. Beliman, "Dynamic Programming," Princeton Univ. Press, Princeton, N.J., 1957. 
16. V. G. Baltyanskil, R. V. Gamkrelidze, and L. S. Pantryagin, "Dn the Theory of Optimel Pracesses," Izv. Akad. Nauk 555R, vol.24, pp. 3-42, 1960.

17. M. Athans and P. Falb, "Optimal Contral," McGraw-Hill Book Co., Inc, New Yark, N.Y., 1966.

18. A. E. Bryson, Jr., and Y. C. Ha, "Applied Dptimal Control," Blaisdell Co., Waltham, Mass. 1969.

19. R. Bellman, "A Markoffian Decision Process," Jour. of Math. and Mechanics, vol.6, pp.679-684, 1957.

20. R. A. Howard, "Dynamic Programming and Markoff Processes," M.I.T. Press and J. Wiley, Inc., Cambridge, Mass. and New York, N.Y., 1960.

21. P. Walfe and G. B. Dantzig, "Linear Programming in a Markaff Chain," Oper. Res., vol.10, pp.702-710, 1962.

22. C. Derman and M. Klein, "Some Remarks on Finite Horizon Markoffian Decision Models," Dperations Research, vol.13, pp.272-278, 1965.

23. C. Derman, "Markaffian Sequential Contral Processes, - Denumerable State Space," Jour. Math. Anal. and Appl., vol.10, pp.295-302, 1965. 
24. D. Blackwell, "Discounted Dynamic Programming," Anr. Math. Stat., vol.36, pp.226-235, 1965.

25. A. F. Veinott, Jr., "On Finding Optimal Policies in Discrete Dynamic Programming with no Discounting," Ann. Math. Stat., vol.37, pp.1284-1294, 1966.

26. R. M. Karp and M. Held, "Finite-State Processes and Dynamic Programming," SIAM Jour. an Appl. Math., vol.15, pp.693-718, 1967.

27. E. V. Denarda, "Contraction Mappings in the Theory Underlying Dynamic Programming," SIAM Review, vol.9, pp.165-177, 1967. 


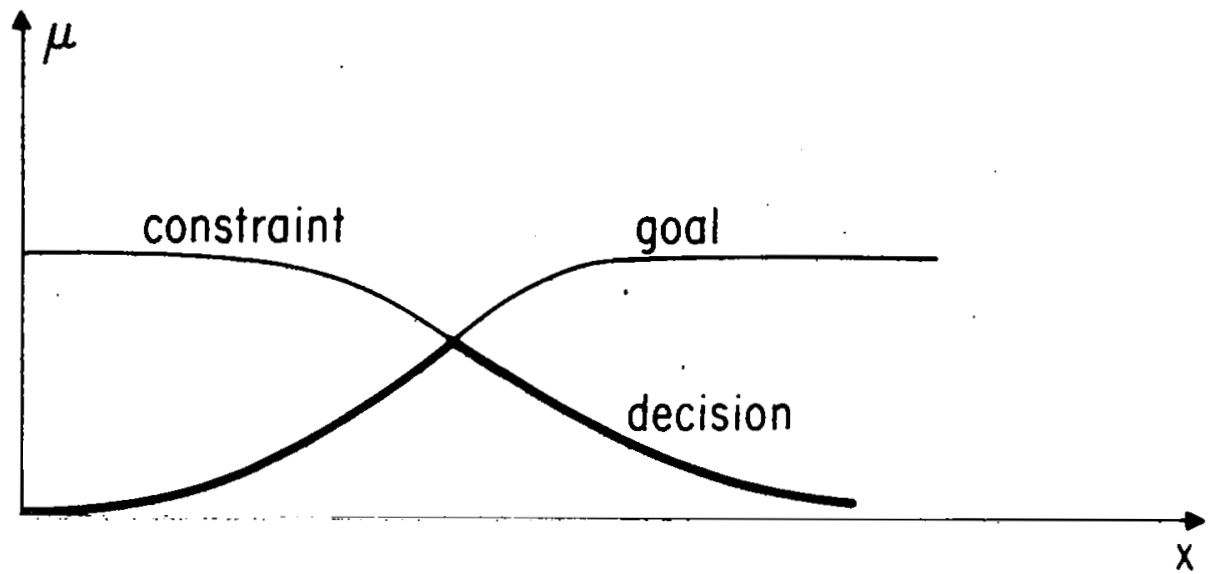

Fig. 1. Relation between the goal, the constraint and the decision. 


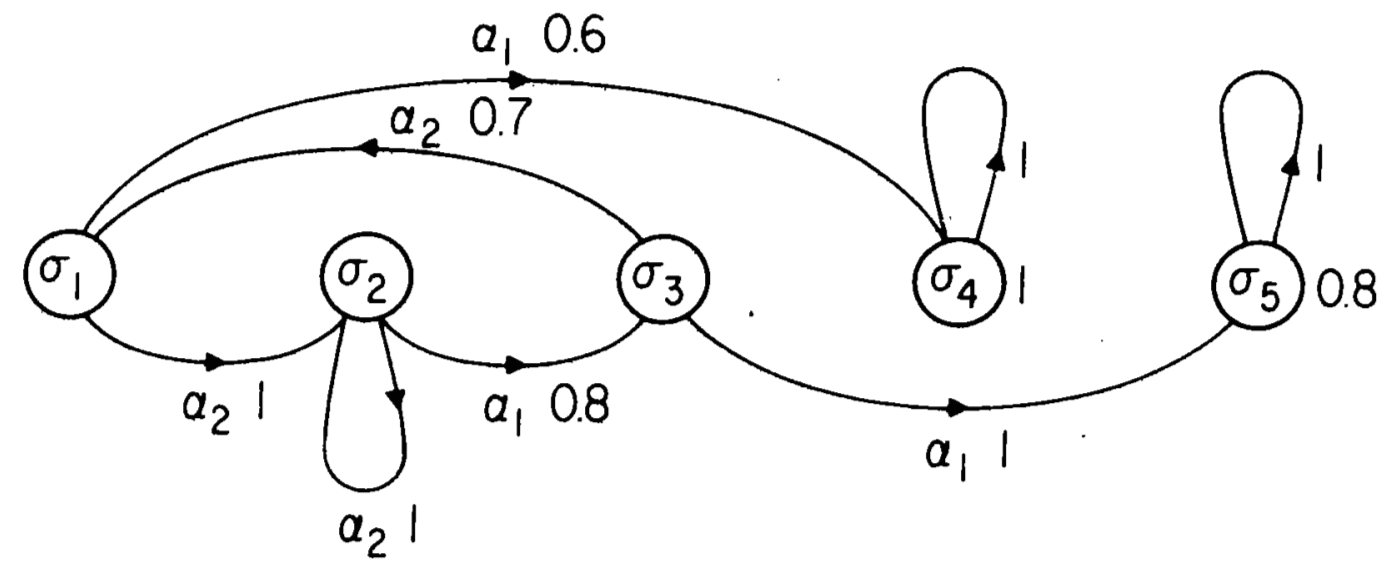

Fig. 2. State diagram for A. 


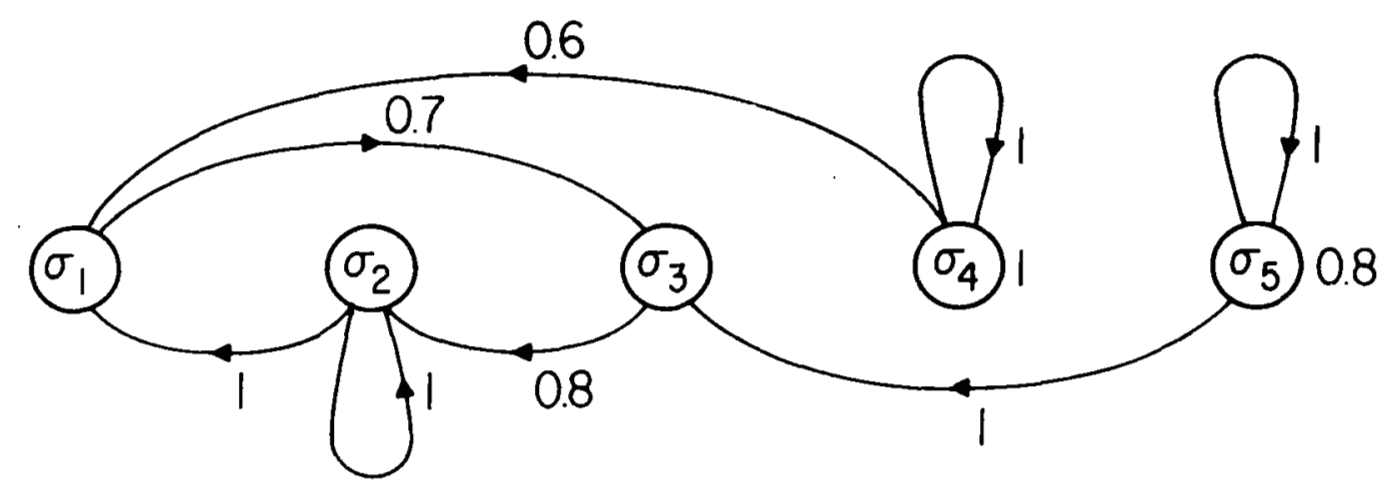

Fig. 3. Reversed-flow state diagram 